\title{
Impact of sleep disturbances on kidney function decline in the elderly
}

\author{
Isabelle Jaussent ${ }^{1,2}$, Jean-Paul Cristol ${ }^{2,3,4}$, Benedicte Stengel ${ }^{5,6,7}$, \\ Marie-Laure Ancelin 1,2, Anne-Marie Dupuy 1,2,3, Alain Besset ${ }^{1,2}$, \\ Catherine Helmer ${ }^{8,9}$, Karen Ritchie ${ }^{1,2,10}$, Claudine Berr ${ }^{1,2}$ and Y'ves Dauvilliers ${ }^{1,2,11}$ \\ Affiliations: ${ }^{1}$ Inserm, U1061, Montpellier, France. ${ }^{2}$ Université Montpellier, Montpellier, France. ${ }^{3}$ Laboratoire de \\ Biochimie, CHRU de Montpellier, Montpellier, France. ${ }^{4}$ Inserm, U1046, CNRS UMR 9214, Montpellier, France. \\ ${ }^{5}$ Inserm U1018, Team 5, Villejuif, France. ${ }^{6}$ Université Paris-Sud, Villejuif, France. ${ }^{7}$ Université Versailles-Saint- \\ Quentin, France. ${ }^{8}$ Inserm, U897, Bordeaux, France. ${ }^{9}$ Université de Bordeaux, ISPED, Bordeaux, France. \\ ${ }^{10}$ Faculty of Medicine, Imperial College, London, UK. ${ }^{11}$ Service de Neurologie, Unité des Troubles du Sommeil, \\ CHU Montpellier, Hôpital Gui-de-Chauliac, Montpellier, France.
}

Correspondence: Isabelle Jaussent, Inserm-U1061, Hôpital La Colombiere, 39, Avenue Charles Flahault, BP 34493, 34093 Montpellier Cedex 5, France. E-mail: isabelle.jaussent囚inserm.fr

ABSTRACT While sleep disturbances are frequent in renal disease patients, no studies have examined prospectively the associations between sleep disturbances and kidney function decline in communitydwelling elderly subjects.

Glomerular filtration rates (eGFRs) were estimated at baseline and at 11-year follow-up. A glomerular filtration decline over the follow-up period was defined as a percentage decline greater than or equal to the cut-off value of the highest tertile of kidney function decline (22\%) in 1105 subjects. Excessive daytime sleepiness (EDS) and insomnia complaints were self-rated at baseline. Restless legs syndrome (RLS) and its age at onset were assessed at study end-point. An ambulatory polysomnography recording was performed during the follow-up in 277 subjects. Apnoea-hypopnoea index (AHI), periodic limb movements during sleep (PLMS) and total sleep time were analysed.

An increased risk of eGFR decline was associated with EDS (OR 1.67, 95\% CI 1.18-2.34) and RLS (OR 1.98, 95\% CI 1.18-3.30) independently of potential confounders including cardiovascular risk factors. Among insomnia complaints, a borderline association with eGFR decline was found for early morning awakening only. High AHI $\left(\geqslant 30\right.$ events. $\left.h^{-1}\right)$ and short total sleep time $(<6 \mathrm{~h})$, but not PLMS were linked to eGFR decline in crude associations, but only AHI remained significantly associated after multi-adjustments.

EDS, RLS and AHI constitute independent risk factors for kidney glomerular function decline.

This article has supplementary material available from erj.ersjournals.com

Received: July 162015 | Accepted after revision: Oct 03 2015 | First published online: Dec 032015

Support statement: The 3C Study is conducted under a partnership agreement between Inserm, the Victor Segalen Bordeaux II University and Sanofi-Synthélabo. The Foundation for Medical Research funded the preparation and first phase of the study. The 3C Study is also supported by the French National Fund for Health Insurance of Employees (CNAMTS), the General Directorate of Health (DGS), MGEN, the Institute of Longevity, the French Agency for Safety of Health Products (AFSSPS), the regional governments of Aquitaine, Bourgogne and Languedoc-Roussillon and the Fondation of France, the Ministry of Research-Inserm programme "Cohorts and collection of biological material". The Lille Génopôle received an unconditional grant from Eisai. The Fondation Plan Alzheimer funded the follow-up of the cohort. Part of this project is financed by grants from the French National Research Agency (projects ANR 2007-LVIE-004 and 06-PNRA-005).

Conflict of interest: Disclosures can be found alongside the online version of this article at erj.ersjournals.com

Copyright OERS 2016 


\section{Introduction}

Sleep disturbances and chronic kidney disease (CKD) are often comorbid and are both common conditions in the elderly. Their prevalence increases with age [1,2]. Sleep disturbances such as insomnia, sleep apnoea syndrome, restless legs syndrome (RLS) and excessive daytime sleepiness (EDS) are very common in patients with early or late end-stage renal disease [3, 4]. However, it remains unclear whether the associations between sleep disorders and CKD are unidirectional or bidirectional.

Few longitudinal studies on sleep disorders and renal function have been conducted in solely clinical populations. One study reported that patients with various degrees of chronic renal failure had progressively worse sleep quality over a 3-year follow-up [5]. Conversely, another study reported that patients newly diagnosed with CKD improved their sleep quality over a 4-year period [6]. These inconstancies could be attributed to differences in design, sample size, clinical setting and heterogeneity in CKD stages. The impact of sleep disorders on the development and progression of cardiovascular disease, hypertension, diabetes and obesity has been previously observed [7,8], all factors associated with decline in glomerular filtration rate (GFR) [9]. It has been suggested that sleep fragmentation due to periodic limb movements during sleep (PLMS), respiratory events and pain may induce a renin-angiotensin-aldosterone system hyperactivation, autonomic nervous system dysregulation and consequent increases in blood pressure which in turn increase risk of CKD progression. High rates of insomnia and EDS complaints in the CKD population may also contribute to the burden of cardiovascular disease in this at-risk population, as already reported in the general population $[10,11]$.

In view of these conflicting results, further prospective studies are required to better understand the directionality of these associations, notably to determine whether sleep disturbances aggravate CKD or if CKD increases the severity of sleep problems per se. So far, no studies have assessed prospectively whether sleep disturbances alter kidney function in the general elderly population. We thus proposed to assess, over an 11-year follow-up, the relationships between insomnia complaints, EDS, sleep apnoea syndrome, RLS and glomerular filtration decline in community-dwelling elderly people, taking into account confounding factors comorbid with renal alteration.

\section{Methods}

\section{Study population}

Participants were recruited as part of the Three-City (3C) Study, an ongoing multisite longitudinal study involving three French cities: Bordeaux, Dijon and Montpellier. Briefly, noninstitutionalised participants aged $\geqslant 65$ years were randomly selected from the electoral rolls between 1999 and 2001 . The study protocol was approved by the ethical committee of the University Hospital of Kremlin-Bicêtre (Paris, France) and the Comité de Protection des Personnes Sud Méditéranée III (Nimes, France), and written informed consent was obtained from each participant. The participants were administered standardised questionnaires and underwent clinical examinations at baseline and at 2-, 4-, 8- and 11-year follow-up. Serum creatinine measurement was performed at baseline and at 11-year follow-up in the Montpellier and Bordeaux centres only.

\section{Assessment of change in GFR}

Serum creatinine was measured at a single laboratory using the colometric Jaffe method at both timepoints. To standardise creatinine values, 1720 frozen serum baseline samples from all three centres [12] and 301 from the Montpellier centre at end-point were remeasured using an isotope dilution mass spectrometry traceable enzymatic assay (Roche assay) [13]. Equations were developed at both times to standardise the results obtained from the Jaffe assay to enzymatic measurements. GFR expressed as $\mathrm{mL} \cdot \mathrm{min}^{-1} \cdot 1.73 \mathrm{~m}^{-2}$ was estimated (eGFR) using the Chronic Kidney Disease Epidemiology Collaboration formula without correction for ethnicity (not available in the 3C Study and not recommended in France by the French National Authority for Health) [14]. At baseline, eGFR categories were defined according to the National Kidney Foundation guidelines [15] as 1) stages 1-2: normal to mild decrease, eGFR $\geqslant 60 \mathrm{~mL} \cdot \mathrm{min}^{-1} \cdot 1.73 \mathrm{~m}^{-2}$; 2) stage 3 : moderate alteration, eGFR $30-59 \mathrm{~mL} \cdot \mathrm{min}^{-1} \cdot 1.73 \mathrm{~m}^{-2}$; and 3) stage 4 or higher: severe alteration, eGFR $<30 \mathrm{~mL} \cdot \mathrm{min}^{-1} \cdot 1.73 \mathrm{~m}^{-2}$. Percentage change in eGFR over time was calculated as: (baseline eGFR - 11-year eGFR)/(baseline eGFR) $\times 100$. A significant eGFR decline $\geqslant 22 \%$ (highest tertile of eGFR change) was used to study kidney function decline in our cohort.

\section{Sleep assessment}

Sleep complaints were assessed at baseline as part of a clinical interview performed by a psychologist or nurse, followed by the self-completion of a sleep questionnaire. The participants were invited to answer never, rarely, frequently or often to the following questions: "Do you feel very sleepy during the day?" (EDS); "Do you have any difficulty in falling asleep?" (difficulty in initiating sleep); "Do you wake up 
during the night?" (difficulty in maintaining sleep); "Do you often wake up early in the morning without being able to go back to sleep?" (early morning awakening (EMA)); and "Do you snore loudly?"

In the analyses, EDS was defined as reporting being excessively sleepy frequently/often. Insomnia complaints based on difficulty in initiating sleep, difficulty in maintaining sleep and EMA were also dichotomised as frequently/often versus never/rarely and summed to obtain the number of insomnia complaints (range 0-3). The risk of a "potential" sleep apnoea syndrome was defined as being obese (body mass index $(\mathrm{BMI})>30 \mathrm{~kg} \cdot \mathrm{m}^{-2}$ ) with frequent/often loud snoring and EDS.

RLS was assessed at study end-point using the four questions designed to address the minimal diagnostic criteria of the International RLS Study Group [16]. Diagnosis of RLS was based on four positive answers, with a further question asked on age at onset of RLS. We excluded for this analysis 29 participants with Parkinson's disease or treated using dopaminergic therapy.

Among the participants with creatinine measured at both evaluations, 277 (all from the Montpellier centre) underwent, on a voluntary basis, an ambulatory polysomnography (PSG) recording during the follow-up (median (range) 9.7 (4.80-12.20) years). No selection was performed on sleep complaints and renal function. PSG recordings were made in the subjects' home environment using the Deltamed coherence system (Natus, Pleasanton, CA, USA) which includes five electroencephalography leads, right and left electro-oculograms, electromyography of chin and tibialis anterior muscles, electrocardiogram, nasal cannula/pressure transducer, mouth thermistor, chest and abdominal bands, body position and pulse oximeter. Sleep stage, micro-arousals, PLMS and respiratory events were scored manually according to standard criteria [17]. Obstructive apnoea was defined as a complete airflow cessation for $>10 \mathrm{~s}$ associated with thoracoabdominal movements, and hypopnoea as airflow reduction of $>50 \%$ associated with a drop in arterial oxygen saturation measured by pulse oximetry of $>3 \%$ or a micro-arousal. The average number of apnoeas/hypopneas per hour of sleep (apnoea-hypopnoea index (AHI)) was calculated. In the present study, only AHI and its related oxygen saturation parameters (i.e. mean and minimum oxygen saturation, and percentage of time $<90 \%$ ), PLMS and total sleep time (TST) were taken into account, with TST $<6 \mathrm{~h}$, $\mathrm{AHI} \geqslant 30$ events. $\mathrm{h}^{-1}$ and PLMS $\geqslant 15$ events $\cdot \mathrm{h}^{-1}$ considered as pathological conditions.

\section{Assessment of covariates}

The standardised interview at baseline included questions on sociodemographic characteristics, alcohol consumption, caffeine intake and smoking status, health status and medication use. Case-level depressive symptoms were defined as a score $\geqslant 16$ on the Center for Epidemiologic Studies depression scale [18] or currently taking antidepressant treatment. Cognitive impairment was defined as a score $<26$ on the Mini Mental State Examination [19]. Disability was assessed using the Instrumental Activities of Daily Living scale [20]. Anthropometric measurements, including height and weight were performed during the clinical examination to calculate BMI $\left(\mathrm{kg} \cdot \mathrm{m}^{-2}\right)$. Hypertension was defined by measured systolic blood pressure $\geqslant 160 \mathrm{mmHg}$ or diastolic blood pressure $\geqslant 95 \mathrm{mmHg}$ or current antihypertensive treatment. Diabetes was defined as fasting glucose level $\geqslant 7.0 \mathrm{mmol} \cdot \mathrm{L}^{-1}$ or treatment for diabetes, and hypercholesterolaemia as total cholesterol level $\geqslant 6.2 \mathrm{mmol} \cdot \mathrm{L}^{-1}$ or treatment with lipid-lowering agents. History of respiratory and cardio-cerebrovascular diseases (angina pectoris, myocardial infarction, cardiovascular surgery, arteritis and stroke) was investigated. Drugs were coded according to the World Health Organization's Anatomical Therapeutic Chemical classification [21]. Hypnotics were classified as benzodiazepines, benzodiazepine-like compounds (zolpidem and zopiclone) and miscellaneous medications (including barbiturates, antihistamines and other pharmacological categories such as neuroleptics) [22].

\section{Statistical analyses}

Logistic regression models were used to compare the characteristics of participants according to the eGFR categories $\left(<60\right.$ versus $\geqslant 60 \mathrm{~mL} \cdot \mathrm{min}^{-1} \cdot 1.73 \mathrm{~m}^{-2}$ ) at baseline after adjustment for study centre, age and sex. To analyse the associations of eGFR decline ( $\geqslant 22 \%$ versus $<22 \%)$ with sleep disturbances, logistic regression models were used to estimate odds ratios and their $95 \%$ confidence intervals. Multivariate models included study centre and covariates associated with eGFR decline at $\mathrm{p}<0.15$. Where appropriate, the interaction terms were tested using the Wald Chi-squared test. Secondary analyses were implemented in a subsample of subjects free of CKD (i.e. eGFR $\geqslant 60 \mathrm{~mL} \cdot \mathrm{min}^{-1} \cdot 1.73 \mathrm{~m}^{-2}$ ) at baseline and in those with ambulatory PSG, to assess the impact of AHI, PLMS and TST on eGFR decline. The significance level was set at $\mathrm{p}<0.05$. Analyses were performed using SAS version 9.4 (SAS Inc., Cary, NC, USA).

\section{Results}

\section{Subject characteristics}

The final sample included 1105 participants from Bordeaux $(\mathrm{n}=438)$ and Montpellier $(\mathrm{n}=667)$ with a median (range) baseline age of 70.9 (65.0-87.8) years, of whom $57.9 \%$ were female. As detailed in the flow 
chart diagram (fig. 1), these subjects were free of dementia, had fully completed the sleep questionnaire, with eGFRs evaluated at baseline and 11-year follow-up, and without missing data in baseline adjustment covariates. Participants excluded from the study had significantly a lower education level, were older, living alone with activity limitations, more cardiovascular risk factors, chronic disease and sleep disturbances, taking more hypnotics and had a lower baseline eGFR.

At baseline, $19.2 \%$ of the participants had frequent/often EDS and $74.8 \%$ had insomnia complaints, of whom $30.2 \%$ had three insomnia complaints. Furthermore, $17.1 \%$ of the participants used sleep medication regularly $(67.4 \%$ benzodiazepines, $32.1 \%$ benzodiazepine-like compounds and $11.6 \%$ miscellaneous medication). Among those subjects, $45.8 \%$ had three insomnia complaints and $13.7 \%$ had none. RLS was reported by $22.2 \%(\mathrm{n}=196)$ of subjects $(62.9 \%$ with difficulty in maintaining sleep symptoms, $41.4 \%$ with difficulty in initiating sleep and $35.7 \%$ with EMA; and $27.1 \%$ without any insomnia complaints), of whom $35.7 \%$ declared having RLS before study inclusion.

Median (interquartile range (IQR)) baseline eGFR was $81(72-88) \mathrm{mL} \cdot \mathrm{min}^{-1} \cdot 1.73 \mathrm{~m}^{-2}$. Only $9.0 \%(\mathrm{n}=74)$ had a baseline eGFR $<60 \mathrm{~mL} \cdot \mathrm{min}^{-1} \cdot 1.73 \mathrm{~m}^{-2}$, including one participant whose eGFR was $<30 \mathrm{~mL} \cdot \mathrm{min}^{-1} \cdot 1.73 \mathrm{~m}^{-2}$. The participants with $\mathrm{eGFR}<60 \mathrm{~mL} \cdot \mathrm{min}^{-1} \cdot 1.73 \mathrm{~m}^{-2}$ differed significantly from those with an eGFR $\geqslant 60 \mathrm{~mL} \cdot \mathrm{min}^{-1} \cdot 1.73 \mathrm{~m}^{-2}$ in having a greater frequency of hypercholesterolaemia and hypertension after adjustment for centre, age and sex. No significant associations were found between eGFR levels and sleep disturbances (online supplementary table S1).

\section{Association between sleep disturbances and eGFR decline over 11-year follow-up}

The median (range) delay between the collections of biological samples was $11(10.0-12.5)$ years. Over this period, $32.1 \%(n=355)$ had a moderate to severe eGFR decline, including 18 participants with a severe decline in eGFR. The median (IQR) percentage of eGFR decline was $14(7-26) \%$, which corresponds to $1.32(0.59-2.32) \%$ per year.

Baseline sociodemographic and clinical characteristics of participants according to kidney function decline are given in table 1 . Participants with a decline $\geqslant 22 \%$ (i.e. the highest tertile of kidney function decline) were more frequently male, older, overweight or obese, had diabetes mellitus and hypertension. They also tended to be past or current smokers and had a history of cardiovascular disease $(\mathrm{p}<0.10)$. Subsequent analyses were thus adjusted for these factors.

Table 2 shows the associations of baseline sleep disturbances with eGFR decline during the follow-up. Whereas difficulty in initiating sleep, difficulty in maintaining sleep and the number of insomnia complaints were not significantly associated with eGFR decline, a borderline association was observed for EMA after adjustment for potential confounders (model 2; OR 1.32, 95\% CI 0.99-1.75). The risk of eGFR decline increased with loud snoring (model 2; OR 1.37, 95\% CI 1.04-1.81) and clinically defined apnoea (model 2; OR 2.59, 95\% CI 1.16-5.78). A positive association was also observed between EDS and eGFR decline even after multiple adjustments (model 2; OR 1.67, 95\% CI 1.18-2.34) and persists even after further adjustment for loud snoring and EMA (OR 1.58, 95\% CI 1.12-2.23; $\mathrm{p}=0.009$ ). No significant interactions were found for eGFR decline between EDS and sex or age.

RLS also increased the risk of eGFR decline when its age at onset preceded study inclusion (model 2; OR 1.98, 95\% CI 1.18-3.30), even after adjustment for EDS (OR 1.90, 95\% CI 1.13-3.18; p=0.02). In contrast, no significant increased risk of eGFR decline was found when RLS was reported at end-point only.

FIGURE 1 Flow chart of participant inclusion.
4216 free of dementia included in the Three-city Study Bordeaux and Montpellier centres

245 without serum creatinine at baseline

885 with incomplete sleep questionnaires

161 with missing data in adjustment covariables

2925 included

599 deaths

640 without follow-up at 11 years

581 without serum creatinine at 11 years

1105 included in the final sample 
TABLE 1 Baseline sociodemographic and clinical characteristics of participants according to the percentage decline in estimated glomerular filtration rate (eGFR) over the 11-year follow-up

\begin{tabular}{|c|c|c|c|c|}
\hline & \multicolumn{4}{|c|}{ eGFR decline } \\
\hline & $<22 \%$ & $\geqslant 22 \%$ & OR $(95 \% \mathrm{CI})$ & p-value \\
\hline Subjects $n$ & 736 & 369 & & \\
\hline \multicolumn{5}{|l|}{ Age years } \\
\hline$<68.92$ & $267(36.28)$ & $101(27.37)$ & 1 & \multirow[t]{3}{*}{0.0004} \\
\hline $68.92-72.99$ & $251(34.10)$ & $117(31.71)$ & $1.23(0.90-1.69)$ & \\
\hline$\geqslant 72.99$ & $218(29.62)$ & $151(40.92)$ & $1.83(1.34-2.49)$ & \\
\hline \multicolumn{5}{|l|}{ Sex } \\
\hline Male & $293(39.81)$ & $172(46.61)$ & 1 & \multirow[t]{2}{*}{0.03} \\
\hline Female & $443(60.19)$ & 197 (53.39) & $0.76(0.59-0.97)$ & \\
\hline \multicolumn{5}{|c|}{ High educational level } \\
\hline No & $531(72.15)$ & $280(75.88)$ & 1 & \multirow[t]{2}{*}{0.19} \\
\hline Yes & 205 (27.85) & 89 (24.12) & $0.82(0.62-1.10)$ & \\
\hline \multicolumn{5}{|c|}{ Activity limitations: IADL } \\
\hline No & 710 (96.47) & $353(95.66)$ & 1 & \multirow[t]{2}{*}{0.51} \\
\hline Yes & 26 (3.53) & $16(4.34)$ & $1.24(0.66-2.34)$ & \\
\hline \multicolumn{5}{|l|}{ Living alone } \\
\hline No & $557(75.68)$ & $286(77.51)$ & $1.11(0.82-1.49)$ & \multirow[b]{2}{*}{0.50} \\
\hline Yes & 179 (24.32) & 83 (22.49) & 1 & \\
\hline \multicolumn{5}{|l|}{ MMSE score } \\
\hline$\geqslant 26$ & $667(90.63)$ & $330(89.43)$ & 1 & \multirow[t]{2}{*}{0.53} \\
\hline$<26$ & 69 (9.38) & $39(10.57)$ & $1.14(0.75-1.73)$ & \\
\hline \multicolumn{5}{|c|}{ Depressive symptoms } \\
\hline No & 599 (81.39) & 289 (78.32) & 1 & \multirow[t]{2}{*}{0.23} \\
\hline Yes & $137(18.61)$ & $80(21.68)$ & $1.21(0.89-1.65)$ & \\
\hline \multicolumn{5}{|l|}{$\mathrm{BMI} \mathbf{k g} \cdot \mathrm{m}^{-2}$} \\
\hline$<25$ & 397 (53.94) & 150 (40.65) & 1 & 0.0001 \\
\hline $25-30$ & 274 (37.23) & $171(46.34)$ & $1.65(1.26-2.16)$ & \\
\hline$\geqslant 30$ & 65 (8.83) & $48(13.01)$ & 1.95 (1.29-2.97) & \\
\hline Caffeine intake & & & & \\
\hline$\leqslant 125$ & 167 (22.69) & $98(26.56)$ & 1 & 0.27 \\
\hline $125-375$ & 409 (55.57) & $202(54.74)$ & $0.84(0.62-1.14)$ & \\
\hline$>375$ & $160(21.74)$ & $69(18.70)$ & $0.73(0.50-1.07)$ & \\
\hline Alcohol g.day & & & & \\
\hline 0 & 99 (13.45) & $53(14.36)$ & 1 & 0.15 \\
\hline $1-36$ & 568 (77.17) & 268 (72.63) & $0.88(0.61-1.27)$ & \\
\hline$\geqslant 36$ & 69 (9.38) & $48(13.01)$ & $1.30(0.79-2.14)$ & \\
\hline Smoking status & & & & \\
\hline Never & $454(61.68)$ & $202(54.74)$ & 1 & 0.08 \\
\hline Past & 245 (33.29) & 144 (39.02) & $1.32(1.01-1.72)$ & \\
\hline Current & 37 (5.03) & $23(6.23)$ & $1.40(0.81-2.41)$ & \\
\hline Diabetes mellit & & & & \\
\hline No & 696 (94.57) & 332 (89.97) & 1 & 0.005 \\
\hline Yes & $40(5.43)$ & $37(10.03)$ & $1.94(1.22-3.09)$ & \\
\hline Hypercholester & & & & \\
\hline No & $474(64.40)$ & 246 (66.67) & 1 & 0.46 \\
\hline Yes & $262(35.60)$ & 123 (33.33) & $0.90(0.69-1.18)$ & \\
\hline Hypertension & & & & \\
\hline No & 409 (55.57) & $153(41.46)$ & 1 & $<0.0001$ \\
\hline Yes & 327 (44.43) & 216 (58.54) & 1.77 (1.37-2.27) & \\
\hline Respiratory dis & & & & \\
\hline No & 702 (95.38) & 352 (95.39) & 1 & 0.99 \\
\hline Yes & 34 (4.62) & $17(4.61)$ & $1.00(0.55-1.81)$ & \\
\hline History of cardi & & & & \\
\hline No & 593 (80.57) & 279 (75.61) & 1 & 0.06 \\
\hline Yes & $143(19.43)$ & 90 (24.39) & 1.34 (0.99-1.81) & \\
\hline Hypnotic use & & & & \\
\hline No & 613 (83.29) & $302(81.84)$ & 1 & 0.55 \\
\hline Yes & $123(16.71)$ & $67(18.16)$ & $1.11(0.80-1.53)$ & \\
\hline
\end{tabular}

Data are presented as $\mathrm{n}(\%)$, unless otherwise stated. IADL: Instrumental Activities of Daily Living; MMSE: Mini Mental State Examination; BMI: body mass index. 
TABLE 2 Baseline sleep complaints of participants according to the percentage decline in estimated glomerular filtration rate (eGFR) decline over the 11-year follow-up

\begin{tabular}{|c|c|c|c|c|c|c|c|c|}
\hline & \multicolumn{2}{|c|}{ eGFR decline } & \multicolumn{2}{|c|}{ Model $0^{\#}$} & \multicolumn{2}{|c|}{ Model $1^{\pi}$} & \multicolumn{2}{|c|}{ Model $2^{+}$} \\
\hline & $<22 \%$ & $\geqslant 22 \%$ & OR $(95 \% \mathrm{Cl})$ & p-value & OR $(95 \% \mathrm{CI})$ & $\mathrm{p}$-value & OR $(95 \% \mathrm{CI})$ & p-value \\
\hline Subjects $n$ & 736 & 369 & & & & & & \\
\hline \multicolumn{9}{|l|}{ Difficulties in initiating sleep } \\
\hline Never/rarely & $442(60.05)$ & $217(58.81)$ & 1 & 0.69 & 1 & 0.32 & 1 & 0.25 \\
\hline Frequently/often & $294(39.95)$ & $152(41.19)$ & $1.05(0.82-1.36)$ & & $1.15(0.87-1.52)$ & & $1.18(0.89-1.57)$ & \\
\hline \multicolumn{9}{|l|}{$\begin{array}{l}\text { Difficulties in } \\
\text { maintaining sleep }\end{array}$} \\
\hline Frequently/often & 468 (63.59) & $228(61.79)$ & $0.93(0.72-1.20)$ & & $0.91(0.70-1.19)$ & & $0.90(0.69-1.18)$ & \\
\hline \multicolumn{9}{|l|}{ Early morning awakening } \\
\hline Never/rarely & 469 (63.72) & $217(58.81)$ & 1 & 0.11 & 1 & 0.05 & 1 & 0.05 \\
\hline Frequently/often & $267(36.28)$ & $152(41.19)$ & $1.23(0.95-1.59)$ & & $1.32(1.00-1.74)$ & & $1.32(0.99-1.75)$ & \\
\hline \multicolumn{9}{|l|}{$\begin{array}{l}\text { Number of insomnia } \\
\text { complaints }\end{array}$} \\
\hline 0 & $187(25.41)$ & $91(24.66)$ & 1 & 0.87 & 1 & 0.61 & 1 & 0.49 \\
\hline Never/rarely & $615(83.56)$ & $278(75.34)$ & 1 & 0.001 & 1 & 0.0007 & 1 & 0.003 \\
\hline Frequently/often & $121(16.44)$ & $91(24.66)$ & $1.66(1.22-2.26)$ & & $1.78(1.27-2.49)$ & & $1.67(1.18-2.34)$ & \\
\hline \multicolumn{9}{|l|}{ Snoring loudly } \\
\hline Never/rarely & $432(58.70)$ & $184(49.86)$ & 1 & 0.005 & 1 & 0.002 & 1 & 0.02 \\
\hline Frequently/often & $304(41.30)$ & $185(50.14)$ & $1.43(1.11-1.84)$ & & $1.53(1.17-2.00)$ & & $1.37(1.04-1.81)$ & \\
\hline \multicolumn{9}{|l|}{$\begin{array}{l}\text { RLS retrospectively } \\
\text { defined at baseline }\end{array}$} \\
\hline No & $532(93.66)$ & $244(87.77)$ & 1 & 0.004 & 1 & 0.004 & 1 & 0.009 \\
\hline Yes & $36(6.34)$ & $34(12.23)$ & $2.06(1.26-3.37)$ & & $2.07(1.26-3.42)$ & & $1.98(1.18-3.30)$ & \\
\hline \multicolumn{9}{|l|}{ Clinically defined apnoea } \\
\hline No & 724 (98.37) & 354 (95.93) & 1 & 0.02 & 1 & 0.01 & 1 & $0.02^{\S}$ \\
\hline Yes & $12(1.63)$ & $15(4.07)$ & $2.56(1.18-5.52)$ & & $2.82(1.28-6.22)$ & & $2.59(1.16-5.78)$ & \\
\hline
\end{tabular}

Data are presented as n (\%), unless otherwise stated. RLS: restless legs syndrome. \#: crude associations; " : adjusted for centre, age and sex; +: adjusted for all the covariates on model 1 plus body mass index (BMI), smoking status, diabetes mellitus, hypertension and history of cardiovascular disease; ${ }^{\S}$ : for apnoea, the adjustment for BMI was not applied because of colinearity.

\section{Sensitivity analyses}

Supplementary analyses were performed: 1) excluding the 74 participants with moderate to severe eGFR impairment $\left(<60 \mathrm{~mL} \cdot \mathrm{min}^{-1} \cdot 1.73 \mathrm{~m}^{-2}\right)$ at baseline; and 2) taking into account the new cerebro-cardiovascular events reported over the follow-up $(n=71)$; and the results remained unchanged.

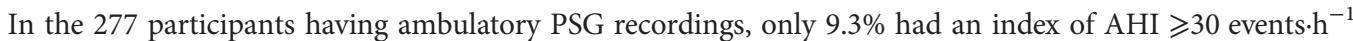
and this was associated with loud snoring $(\mathrm{p}=0.007)$, but not with EDS $(\mathrm{p}=0.90)$ or BMI $(\mathrm{p}=0.22)$. A high AHI was also associated with eGFR decline (OR 2.80, 95\% CI 1.21-6.44; $\mathrm{p}=0.02$ ) and the results remained significant after adjustments for age, sex, BMI, smoking status, diabetes mellitus, hypertension, history of cardiovascular disease and EDS (OR 2.50, 95\% CI 1.01-6.20; $\mathrm{p}=0.04$ ) (data not shown). No significant interaction was found between AHI and EDS for eGFR decline $(p=0.99)$. Furthermore, no significant associations were found either for mean $(\mathrm{p}=0.34)$ or minimum oxygen saturation $(\mathrm{p}=0.16)$ or for percentage of time $<90 \%(p=0.28)$. A short TST $(<6 \mathrm{~h})$ was found in $50.4 \%$ of subjects, without any association with insomnia complaints. A short TST was associated with an eGFR decline (OR 1.75, 95\% CI 1.05-2.93; $\mathrm{p}=0.03)$; however the results became nonsignificant after multi-adjustments $(\mathrm{p}=0.08)$. A PLMS index $\geqslant 15$ events. $\mathrm{h}^{-1}$, found in $62.4 \%$ of subjects, was associated with RLS at end-point only ( $\left.\mathrm{p}=0.04\right)$, but not when already reported at study inclusion $(p=0.10)$. Finally, no significant associations were found between PLMS and eGFR decline in neither the crude $(\mathrm{p}=0.20)$ nor in adjusted associations $(\mathrm{p}=0.40)$.

\section{Discussion}

We examined associations between a large range of sleep disturbances and eGFR decline at 11-year follow-up in a general elderly population. Subjects with EDS had a 1.7 -fold greater risk of having eGFR decline after adjustment for numerous potential confounders, including baseline cardiovascular risk factors. 
RLS was also found to be associated with a two-fold higher risk of eGFR decline independently of EDS. With the exception of EMA, which showed a borderline association, insomnia complaints were not related to eGFR decline. In a subsample, high AHI and shorter TST, but not PLMS were linked to eGFR decline in crude associations. Only AHI remained significant after adjustments.

Although associations between renal function and sleep have been observed previously [4], to our knowledge no study has investigated the impact over time of sleep disturbances on eGFR decline in a general population sample. The few clinical studies that examined the relationship between sleep quality and renal failure in CKD patients with early or end-stage disease have produced conflicting results $[5,6]$. The association between EDS and eGFR decline reported in our study was independent of cardiovascular diseases, which failed to support the hypothesis-mediating effect of cardiovascular diseases in the relationship between EDS and eGFR in the elderly. Several studies have suggested that cardiovascular and kidney diseases are closely linked through multiple interacting effects including neuroendocrine perturbations, fluid dysregulation, inflammation and immune disturbances, resulting in amplification loops defined as cardiorenal syndrome. In addition to cardiovascular diseases, diabetes, hypertension and obesity can participate in the progression of CKD. We report here that EDS is associated with eGFR decline independently of vascular and metabolic risk factors. Conversely, in previous analyses, we reported that EDS increased the risk of both fatal and nonfatal cardiovascular events, whether first events or recurrent over a 6-year follow-up [11]. The underlying pathophysiological mechanism by which EDS interacts with eGFR decline thus appears to be complex and multifactorial.

Sleep disturbances were often associated with activations of the hypothalamic-pituitary-adrenal axis and sympathetic nervous system, as well as chronic inflammation, which may promote a non-dipping pattern, hypertension and subsequently alter renal function [8]. In our sample, the relationship between EDS and eGFR decline was neither modified by BMI nor by the presence of snoring; however, the latter parameters were weaker predictors of sleep apnoea syndrome in the elderly than in middle-aged adults [23]. In the subsample, no associations were found between AHI and EDS or BMI; however, we reported an association between AHI and eGFR decline independently of EDS and cardiovascular risk factors without any impact of nocturnal hypoxia (i.e. no between-group difference for mean and minimum oxygen saturations). Notably, this differs from the direct nocturnal hypoxia effect already reported on the progression of CKD through a renin-angiotensin-aldosterone system hyperactivation [24, 25]. The link between EDS and eGFR decline may involve a shorter nocturnal sleep duration, a condition frequently associated with increased systemic low-grade inflammation that may trigger cardiovascular disease and subsequent CKD [26]. However, the association loses its significance in multivariate models.

The proportion of subjects with RLS ( $8.3 \%$ at baseline) was in the range of previous reported studies in the elderly [27], and it is known to increase with end-stage renal disease [28]. We found that eGFR decline was predicted by RLS at inclusion but not when present at study end-point only, independently of cardio-vascular risk factors and EDS. These findings suggested that RLS could be an early risk factor for eGFR decline through the bias of an association with sleep fragmentation and periodic-related night-time increased blood pressure [29], conditions that are risk factors for renal dysfunctioning. However, no associations were found between eGFR decline and PLMS. Except for a borderline association with EMA, other subtypes of insomnia complaints and depressive symptoms were not significantly associated with eGFR decline. Altogether, these results supported our previous findings that insomnia complaints were not risk factors for cardiovascular diseases [11], insomnia more likely being a consequence of these chronic diseases.

The present study benefits from several strengths, including standardised creatinine measurements which reduced the bias in the estimate eGFR decline, a large sample size, an 11-year follow-up period in elderly subjects not preselected for kidney disease, and a large range of subjective and objective sleep measures.

The present study has some limitations. Selection bias may have resulted from the recruitment of volunteers from electoral rolls and the exclusion of institutionalised elderly people, which limits the extent to which these findings can be generalised to all older adults. The exclusion of subjects due to missing data, loss to follow-up and death also contributed to selection bias. The excluded subjects were older, had more often chronic disorders, a lower baseline eGFR and were more likely to report sleep complaints and to take hypnotics. This progressive selection of the cohort may cause biases which lead to 1) an underreporting of sleep disorder prevalence and kidney failure incidence rate and 2) a modification (to a lesser extent) of the associations between sleep complaints and eGFR decline.

Sleep complaints were self-reported using a short questionnaire used previously [10,11, 22], which, while lacking external confirmation, remains the most common method for initial diagnosis of sleep pathologies in the primary healthcare setting. Unfortunately, validated sleep measures such as the Epworth Sleepiness Scale, which provides a measurement of the subject's general level of daytime sleepiness, were not available for this study. 
Due to the study design and population (large multicentric elderly cohort), it was not possible to perform PSG in the laboratory for the whole sample. PSG was only possible in the centre of Montpellier (one-quarter of the sample); subjects underwent ambulatory PSG on a voluntary basis, without selection of sleep complaints and renal function. PSG was performed under naturalistic conditions by skilled sleep-specialist technologists with only $2 \%$ of recordings being unusable. PSG recordings were made during the follow-up, precluding examination of relationships with baseline sleep complaints. However, the natural course of sleep disturbances is slow in the elderly [30], thus our small subsample with ambulatory PSG recordings helped to further understand the underlying mechanisms involved in the relationships between sleep disturbances and eGFR decline.

Finally, despite extensive adjustments, the possibility remains that unmeasured factors, such as inflammatory biomarkers may also be involved and confound associations.

\section{Conclusion}

The results of this large 11-year prospective study in the elderly showed that EDS, RLS and AHI constitute risk factors of renal function alteration at a very early stage of decline.

\section{References}

1 Coresh J, Selvin E, Stevens LA, et al. Prevalence of chronic kidney disease in the United States. JAMA 2007; 298: 2038-2047.

2 Ohayon MM, Carskadon MA, Guilleminault C, et al. Meta-analysis of quantitative sleep parameters from childhood to old age in healthy individuals: developing normative sleep values across the human lifespan. Sleep 2004; 27: 1255-1273.

3 Plantinga L, Lee K, Inker LA, et al. Association of sleep-related problems with CKD in the United States, 2005-2008. Am J Kidney Dis 2011; 58: 554-564.

4 Turek NF, Ricardo AC, Lash JP. Sleep disturbances as nontraditional risk factors for development and progression of CKD: review of the evidence. Am J Kidney Dis 2012; 60: 823-833.

5 Sabbatini M, Pisani A, Crispo A, et al. Sleep quality in patients with chronic renal failure: a 3-year longitudinal study. Sleep Med 2008; 9: 240-246.

6 De Santo RM, Bilancio G, Santoro D, et al. A longitudinal study of sleep disorders in early-stage chronic kidney disease. J Ren Nutr 2010; 20: S59-S63.

7 Wolk R, Gami AS, Garcia-Touchard A, et al. Sleep and cardiovascular disease. Curr Probl Cardiol 2005; 30: 625-662.

8 Pepin JL, Borel AL, Tamisier R, et al. Hypertension and sleep: overview of a tight relationship. Sleep Med Rev 2014; 18: 509-519.

9 Yu HT. Progression of chronic renal failure. Arch Intern Med 2003; 163: 1417-1429.

10 Blachier M, Dauvilliers Y, Jaussent I, et al. Excessive daytime sleepiness and vascular events: the Three City Study. Ann Neurol 2012; 71: 661-667.

11 Jaussent I, Empana JP, Ancelin ML, et al. Insomnia, daytime sleepiness and cardio-cerebrovascular diseases in the elderly: a 6-year prospective study. PLoS One 2013; 8: e56048.

12 Stengel B, Metzger M, Froissart M, et al. Epidemiology and prognostic significance of chronic kidney disease in the elderly - the Three-City prospective cohort study. Nephrol Dial Transplant 2011; 26: 3286-3295.

13 Piéroni L, Delanaye P, Boutten A, et al. A multicentric evaluation of IDMS-traceable creatinine enzymatic assays. Clin Chim Acta 2011; 412: 2070-2075.

14 Levey AS, Stevens LA, Schmid $\mathrm{CH}$, et al. A new equation to estimate glomerular filtration rate. Ann Intern Med 2009; 150: 604-612.

15 National Kidney Foundation DIGO 2012. Clinical Practice Guideline for the Evaluation and Management of Chronic Kidney Disease. Kidney Int Suppl 2013; 3: 136-150.

16 Allen RP, Picchietti D, Hening WA, et al. Restless legs syndrome: diagnostic criteria, special considerations, and epidemiology. A report from the restless legs syndrome diagnosis and epidemiology workshop at the National Institutes of Health. Sleep Med 2003; 4: 101-119.

17 Iber C, Ancoli-Israel S, Chesson A, et al. The AASM Manual for the Scoring of Sleep and Associated Events: Rules, Terminology, and Technical Specifications. Westchester, IL, American Academy of Sleep Medicine, 2007.

18 Radloff LS. The CES-D scale: a self-report depression scale for research in the general population. Appl Psychol Meas 1977; 1: 385-401.

19 Folstein MF, Folstein SE, McHugh PR "Mini-mental state". A practical method for grading the cognitive state of patients for the clinician. J Psychiatr Res 1975; 12: 189-198.

20 Lawton MP, Brody EM. Assessment of older people: self-maintaining and instrumental activities of daily living. Gerontologist 1969; 9: 179-186.

21 World Health Organization Collaborating Centre for Drug Statistics Methodology. Guidelines for ATC Classification and DDD Assignment. Oslo, World Health Organization, 2000.

22 Jaussent I, Ancelin ML, Berr C, et al. Hypnotics and mortality in an elderly general population: a 12-year prospective study. BMC Med 2013; 11: 212.

23 Young T, Shahar E, Nieto FJ, et al. Predictors of sleep-disordered breathing in community-dwelling adults: the Sleep Heart Health Study. Arch Intern Med 2002; 162: 893-900.

24 Ahmed SB, Ronksley PE, Hemmelgarn BR, et al. Nocturnal hypoxia and loss of kidney function. PLoS One 2011; 6: e19029.

25 Nangaku M. Chronic hypoxia and tubulointerstitial injury: a final common pathway to end-stage renal failure. J Am Soc Nephrol 2006; 17: 17-25.

26 Cappuccio FP, Cooper D, D’Elia L, et al. Sleep duration predicts cardiovascular outcomes: a systematic review and meta-analysis of prospective studies. Eur Heart J 2011; 32: 1484-1492. 
27 Allen RP, Walters AS, Montplaisir J, et al. Restless legs syndrome prevalence and impact: REST general population study. Arch Intern Med 2005; 165: 1286-1292.

28 Unruh ML, Levey AS, D’Ambrosio C, et al. Restless legs symptoms among incident dialysis patients: association with lower quality of life and shorter survival. Am J Kidney Dis 2004; 43: 900-909.

29 Pennestri MH, Montplaisir J, Colombo R, et al. Nocturnal blood pressure changes in patients with restless legs syndrome. Neurology 2007; 68: 1213-1218.

30 Sforza E, Gauthier M, Crawford-Achour E, et al. A 3-year longitudinal study of sleep disordered breathing in the elderly. Eur Respir J 2012; 40: 665-672. 